\title{
$\begin{array}{ll}\text { Research Square } & \text { Preprints are preliminary reports that have not undergone peer review. } \\ \text { They should not be considered conclusive, used to inform clinical practice, } & \text { or referenced by the media as validated information. }\end{array}$
}

\section{Network-Based Inference of Hub Genes In Epithelial Membrane Protein 2-Treated Human RPE cells}

\section{Hua Wan ( $\square$ wanhua2006@njmu.edu.cn )}

Sir Run Run Hospital Nanjing Medical University https://orcid.org/0000-0002-6030-7413

Wei Zhang

Sir Run Run Hospital Nanjing Medical University

\section{Wei Gao}

Sir Run Run Hospital Nanjing Medical University

\section{Zijiao Tao}

Sir Run Run Hospital Nanjing Medical University

\section{Xiang Lu}

Sir Run Run Hospital Nanjing Medical University

\section{Feng Chen}

Nanjing Medical University School of Basic Medical Sciences

\section{Research Article}

Keywords: differentially expressed genes (DEGs), epithelial membrane protein 2 (EMP2), retinal pigment epithelial cells (hRPECs), computational inference, gene expression, activity proteins (APs), regulatory network, systems biology, hub genes (HGs)

Posted Date: July 1st, 2021

DOI: https://doi.org/10.21203/rs.3.rs-487760/v2

License: (c) (1) This work is licensed under a Creative Commons Attribution 4.0 International License. Read Full License 


\section{Abstract}

Backgrounds Identification of hub genes (HGs) using transcriptome data of human retinal pigment epithelial cells (hRPECs) samples treated with epithelial membrane protein 2 (EMP2) was helpful to accurately evaluate the functional relevance of genetic alterations in activity proteins (APs) in these cells.

Results We performed differential expression genes (DEGs) analyses of public RNA-seq transcriptome data of EMP2 treated hRPECs, vector control (VC) and wild type (WT) hRPECs. VIPER (Virtual Inference of Protein activity by Enriched Regulon analysis) was used to convert DEGs outcomes to APs signatures and ARACNE (Algorithm for the Reconstruction of Accurate Cellular Networks) was used to construct transcription regulatory networks (TRNs) to identify hub genes (HGs).

Conclusions In addition to identifying a significant fraction of DEGs among EMP2-OE groups and EMPKD groups when compared to VC or WT groups, respectively, we also accurately inferred aberrant TGNs and found several HGs induced by EMP2-overexpressed hRPECs under hypoxia. Thus, we raised a hypothesis that EMP2 may regulate hRPECs angiogenesis via a PDGFA regulatory network, which would help to understand the complex biology of angiogenesis in EMP2 and hypoxia treated hRPECs.

\section{Background}

Pathologic retinal neovascularization ${ }^{[1]}$ is a potentially blinding consequence seen in many common diseases including diabetic retinopathy ${ }^{2}$, retinopathy of prematurity ${ }^{[3]}$, retinal vascular occlusive diseases $^{[4]}$, and neovascular age-related macular degeneration (AMD ${ }^{[5]}$, among others. The anti-VEGF treatment has been proven to be highly effective. However, effectiveness decreases over time and side effects exist ${ }^{[6,7]}$. Epithelial membrane protein 2 (EMP2) has shown to reasonably modulate activity through neovascularization network in the retinal pigment epithelial cell line ARPE-19 by controlling VEGF expression ${ }^{[8,9]}$. However, hub genes of angiogenesis related with EMP2 and temporal relationships of their interactions need to be further investigated to understand neovascular disease and identify possible new therapeutic targets.

The presence of HGs in angiogenesis has been well-documented, whose coordinated activity within tightly regulated modules is strictly necessary for angiogenic state initiation and maintenance. We performed differential expression genes (DEGs) analyses of public RNA-seq transcriptome data of EMP2 treated hRPECs comparing to vector control (VC) or wild type (WT) hRPECs. Virtual Inference of protein activity by Enriched Regulon analysis (VIPER) was used to convert the DEGs outcomes to activity proteins (APs) signatures and ARACNE algorithm was used to construct transcription regulatory networks (TRNs) and identify some HGs from TRNs.

\section{Results}


All samples had high-quality RNA-seq reads with an average of $96 \%$ reads per sample. Reads with mapping quality score $(\mathrm{MAPQ})<10$ or those with $>5$ mismatches in $100 \mathrm{bp}$ aligned region were discarded.

\section{Differential Expression of DEGs among OE and KD goups}

After low-level processing of the four tumor stage data and control samples, we performed differential expression analysis for each group compared with control samples. Figure 1A-B shows Venn of DEGs in compared groups under hypoxia and normoxia, respectively. Large difference among the 8 groups is visible.

\section{Enrichment analysis of DEGs in EMP2 treated hRPECs}

After identification of 1239 DEGs from 8 comparing groups under hypoxia and normoxia separately, we performed functional enrichment analysis and found that DEGs of OE versus VC and WT groups under hypoxia commonly enriched in positive regulation of cell proliferation, vasculature development, protein kinase B signaling, and negative regulation of platelet activation, and enriched in metabolism and folate biosynthesis pathway, which did not show in $\mathrm{OE}$ groups under normoxia, as well as in KD groups both under hypoxia and normoxia(Fig. 2C-D).

3.Transcriptome regulatory network (TRN) is topologically different among OE and KD groups

We can appreciate that the intersections in OE TRN increased when compared to the KD TRN. The increase of shared links can be clearly appreciated according to networks growing. Besides, we identified different APs in OE-VC group when compared to KD-VC group, as well as in OE-WT group when compared to KD-WT group under hypoxia (Fig. 5). To further elucidate the regulation and interaction relationship, the APs and DEGs network were visualized using Cytoscape 3.7.1.(Fig. 6). A node is either a DEG or an AP. An edge is an AP-AP, AP-DEG or DEG-DEG relationship. Then the ARACNe algorithm was implemented and TRN of each group was categorized into several sub-networks. We found that all TRNs were substantially different among all groups and HGs of each TRN were analyzed. In the hypoxia OE-VC TRN, we got PDGFA, ALDH1L2, BA1AP3, BBOX1, ST3GAL5, ANGPT1 and KAT6A as HGs, which were not found in the normoxia OE-VC TRN, as well as in KD-WT TRN under hypoxia.

\section{Discussion}

Since early 2010s, RNA-sequencing (RNAseq) has been used to profile transcriptome of retinal neovascularization disease. Differential expression genes (DEGs) analysis can straightforwardly analyze a gene expression dataset. Through DEGs analysis, a set of highly up-regulated and down-regulated genes can be identified by comparing two groups of samples (e.g. hypoxia vs. normoxia). However, gene expression is highly dynamic, and the expression quantification may depend on the techniques (e.g. different platforms of microarray, or RNAseq), making the cross-dataset comparison difficult. The activities of hub genes cannot be directly measured by microarray or RNA-seq because these techniques 
only measure RNA expression level and do not consider gene activity changes by post-translational modifications. Fortunately, hub gene activity can be inferred by its regulons through VIPER, by taking DEGs data and an context-specific gene regulatory network (interactome) as inputs ${ }^{[16]}$.

In present study, transcriptome analysis of EMP2 overexpressed and knock-downed RPE cells under hypoxia or normoxia elucidated eight sets of DEGs which has been used to construct TRN along with variant analysis. TRN analyses attempt to simplify the interactions of activity proteins and emphasize their role in EMP2 treated RPE cells. Activity proteins (APs) are often obscured in standard differential expression analyses (such as those including environmental stressors) and indeed many of the APs identified in our network analysis were not significant in the DEGs analysis, highlighting the strength of the network approach. (Fig. 5)

Under hypoxic condition, 21 DEGs were identified in EMP2-OE group when compared to VC group. However, when we used VIPER to clarify APs, several angiogenic and inflammatory factors (PDGFA, ALDH1L2, BAIAP3, BBOX1, ST3GAL5, ANGPT1, KAT6A) were found to be HGs in EMP2-OE groups. PDGFA and ANGPT1 were identified both in DEGs and APs. They shared some signaling pathway, including MAPK, Ras, Rap1 and PI3K-Akt signaling pathway. Platelet derived growth factor (PDGF) was proved to induce the proliferation and migration effects on RPE cells in PVR ${ }^{[17]}$, and has been reported to participate in the pericytes regulated angiogenesis, vessel stabilization, and contribute to the formation of both the blood-brain and blood-retina barriers by regulating pericyte-endothelial cell communication ${ }^{[18]}$. PDGFR $\beta$ plays an essential role in patient vitreous-stimulated contraction of retinal pigment epithelial cells from epiretinal membranes ${ }^{[19]}$. PDGF-B was also proved to promote cell proliferation and angiogenesis by increasing the activity of Src homology 2 domain-containing tyrosine phosphatase 2 (SHP-2) in RMECs ${ }^{[20]}$. However, PDGF had not proved to have correlation with EMP2.

ALDH1L2 (Aldehyde Dehydrogenase 1 Family Member L2) is a protein coding gene which functions as mitochondrial folate enzyme ${ }^{[21]}$ and the maintenance of mitochondrial integrity and energy balance of the cell $^{[22],}$ of which related pathways are one carbon pool by folate and metabolism of water-soluble vitamins and cofactors. ALDH1L2 had been proved to have correlation with EMP2 in breast cancer cells [23], but it was the first time to be identified in EMP2 treated hRPECs. We also found that PDGFA connected with ALDH1L2 by PLSCR4. By this way, PDGFA may mediate accelerated ATP-independent bidirectional trans-bilayer migration of phospholipids upon binding calcium ions, and it will result in a loss of phospholipid asymmetry in the plasma membrane. This process may play a central role in the initiation of fibrin clot formation, in the activation of mast cells as well as in the recognition of apoptotic and injured cells by the reticuloendothelial system. Thus We raise a hypothesis that over-expression of EMP2 would activate PDGFA through ALDH1L2-PLSCR4 pathway by initializing fibrin clot and recognizing apoptosis.

We also identified that BAIAP3(BAI1 Associated Protein 3) had correlation with MMP16 ${ }^{[24]}$. BAIAPS, functioning in endosome to Golgi retrograde transport, may regulate behavior and food intake by 
controlling calcium-stimulated exocytosis of neurotransmitters including NPY and serotonin and hormones like insulin. Such neurotransmitters have been proved to have the ability to degrade various components of the extracellular matrix, such as collagen type III and fibronectin in the pathogenesis of diabetic cataract ${ }^{[25]}$.

ANGPT was also a HG in OE-VC TRN and had been proven to be upregulated and correlated with VEGF in NVMs ${ }^{[26]}$. It also showed some connection with PDGFA through PLXNA1, CLIP3 and KLF7. OTX2 functions as a transcription factor, probably involved in the development of the brain and the sense organs. It was found to be upregulated in EMP2-knockdown mouse embryoid bodies by participant in gastrulation ${ }^{[27}$ and it was identified in this study to have correlation with PDGFA through ST3GAL5.

Nevertheless, we found that the interaction among OE TRNs under hypoxia increased when compared with KD TRNs. The hypothesis is that when EMP2 over-expressed under hypoxia, PDGFA was upregulated and enhanced the interactions with other genes. The cascade interactions among these genes triggered angiogenesis through complex pathways.

This investigation was mainly based on ARACNe algorithm analysis of transcriptome information of hRPECs treated by hypoxia and exogenous EMP2. The TRNs constructed in this paper provides clues of interaction mechanism between these genes, which not only provides theoretical support for revealing the regulatory relationship among them, but also provides candidate genes, i.e., up- and down-regulated pathway genes,transcriptional factors, and hub genes, which could be studied for future SNP discovery work to establish association with different phenotype/traits of interest. We also established a scientific exploration mode for exploring effective targets of anti-neovascularization, which makes the follow-up basic verification and clinical research more reliable. However, the results of bioinformatics analysis ultimately need to be verified by many experiments and combined with clinical practice to carry out gene function and mechanism verification for substantive exploration.

\section{Conclusions}

In summary, we investigated (1) the basic characteristics and landscape of RNA transcriptome in EMP2 and hypoxia-treated hRPECs; (2) the function of EMP2-mRNAs from signaling pathway, biological process, TRNs and HGs; (3)a HGs centered network that might mediate the comprehensive function of EMP2 and serve as potential diagnostic biomarkers under hypoxia. We found that EMP2 over-expressed hRPECs angiogenesis via a PDGFA regulatory network. The research would pave the way for understanding the complex biology of angiogenesis in EMP2 and hypoxia treated hRPECs.

\section{Methods}

\section{Data Availability}

The RNA-seq data profiles were obtained from the NCBI GEO database (https://www.ncbi.nlm.nih.gov/geo/) GSE151610. The dataset contained 24 samples, which contained 
mRNA profiles of hRPECs panel consisting of EMP2 knock-down(KD), EMP2 overexpressed(OE), vector control(VC), and WT (WT) hRPECs under hypoxia or normoxia. This project was approved by the Institutional Review Board of Sir Run Run Hospital of Nanjing Medical University. All procedures of this study complied with the protocol.

\section{Identification of DEGs}

The data set is available at the NCBI Short-Read Archive (SRA), under accession SRA265560. The RNASeq data set was mapped in the Homo sapiens (human) genome assembly GRCh38 (hg38) by Hisat2. Feature Counts was used in read counts. After filtering all 0 expression genes, DESeq2 R package was used in different expression gene counting. Cutoff value was absolute $\log 2 \mathrm{FC}>1$ and $\mathrm{P}$ value $<0.05$.

\section{GO and KEGG pathway enrichment analysis}

We performed functional enrichment analysis using Kyoto Encyclopedia of Genes, Genomes (KEGG) pathway and Gene Ontology (GO) with a p value $<0.05$ as the significance cutoff. The RichR (https://github.com/hurlab/RichR) package was used in enrichment analysis. (Figure S1)

\section{Activity proteins (APs) inference}

The Virtual Inference of Protein activity by Enriched Regulon analysis (VIPER)[10-12] algorithm was proven to be a useful tool for estimating regulator activity from gene expression data using enriched regulon analysis. VIPER R package was used to compare APs in each group. With the constructed regulatory networks, we computed the activity of each regulator (TFs, coTFs, and surface proteins) using the VIPER program. A set of regulators with significant activity difference were defined at adjusted $p$ value $<0.05$.

\section{Transcription regulatory network construction}

Regulatory networks were constructed by ARACNe (Algorithm for the Reconstruction of Accurate Cellular Networks)[13-15] using both significant APs and significant DEGs and HGs were screened. Cytoscape software (version: 3.8.0) was used for visualizing the regulatory networks. The normalized data were summed after preprocessing and log10 transformed to generate each sample. ARACNe was run with 100 bootstrap iterations using RNA-seq data mapping to a set of 1,239 transcriptional factors (TFs). Parameters were set to zero DPI (Data Processing Inequality) tolerance and MI (Mutual Information) pvalue threshold of $10-8$.

\section{Statistical test}

All statistical tests and plotting were performed in R 3.5.2 (https://www.r-project.org/) unless otherwise mentioned.

\section{Abbreviations}


HGs: hub genes

EMP2: epithelial membrane protein 2

hRPECs: human retinal pigment epithelial cells

APs: activity proteins

DEGs: differential expression genes

VC: victor control

WT: wild type

ARACNE: Algorithm for the Reconstruction of Accurate Cellular Networks

VIPER: Virtual Inference of Protein activity by Enriched Regulon analysis

TRNs: transcription regulatory networks

HGs: hub genes

AMD: age-related macular degeneration

OE: overexpressed

KD: knock-down

SRA: Short-Read Archive

KEGG: Kyoto Encyclopedia of Genes, Genomes

GO: Gene Ontology

\section{Declarations}

\section{Ethics approval and consent to participate}

Not applicable.

\section{Consent for publication}

Not applicable

\section{Availability of data and materials}


The datasets analysed during the current study are available in the NCBI GEO database (https://www.ncbi.nlm.nih.gov/geo/) GSE151610.

\section{Competing interests}

The authors report no conflicts of interest. The authors alone are responsible for the content and writing of this article.

\section{Funding}

This work was funded, in part, by the grant National Natural Science Foundation of China (No. 81922041, No. 81772020, No.81570378) and the Jiangsu Government Scholarship for Overseas Studies [number JS-2018-082].

\section{Authors' contributions}

Author HW and author WZ have given substantial contributions to the conception or the design of the manuscript, author WG and author FC to acquisition, analysis and interpretation of the data. All authors have participated to drafting the manuscript, author $\mathrm{XL}$ revised it critically. All authors read and approved the final version of the manuscript.

\section{Acknowledgments}

The authors acknowledge all the members of their respective teams, from Sir Run Run Hospital, Nanjing Medical University, as well as their collaborators (fundamentalist and clinicians), for their support and interaction during many years of involvement with genetics.

\section{References}

1. Plastino F, Pesce NA, André H. MicroRNAs and the HIF/VEGF axis in ocular neovascular diseases. Acta Ophthalmol 2021; :aos.14845.

2. Yu G, Aaberg MT, Patel TP, lyengar RS, Powell C, Tran A, Miranda C, Young E, Demetriou K, Devisetty L, et al. Quantification of Retinal Nonperfusion and Neovascularization with Ultrawidefield Fluorescein Angiography in Patients With Diabetes and Associated Characteristics of Advanced Disease. JAMA Ophthalmol 2020; 138:680.

3. Aouiss A, Anka Idrissi D, Kabine M, Zaid Y. Update of inflammatory proliferative retinopathy: Ischemia, hypoxia and angiogenesis. Current Research in Translational Medicine 2019; 67:62-71.

4. Esmaili DD, Boyer DS. Recent advances in understanding and managing retinal vein occlusions. F1000Res 2018; 7:467. 
5. Kiss S, Malangone-Monaco E, Wilson K, Varker H, Stetsovsky D, Smith D, Garmo V. Real-World Injection Frequency and Cost of Ranibizumab and Aflibercept for the Treatment of Neovascular Age-Related Macular Degeneration and Diabetic Macular Edema. JMCP 2020; 26:253-66.

6. Campochiaro PA, Akhlaq A. Sustained suppression of VEGF for treatment of retinal/choroidal vascular diseases. Progress in Retinal and Eye Research 2020; :100921.

7. Ngo Ntjam N, Thulliez M, Paintaud G, Salvo F, Angoulvant D, Pisella P-J, Bejan-Angoulvant T. Cardiovascular Adverse Events with Intravitreal Anti-Vascular Endothelial Growth Factor Drugs: A Systematic Review and Meta-analysis of Randomized Clinical Trials. JAMA Ophthalmol [Internet] 2021 [cited 2021 Apr 30]; Available from:

https://jamanetwork.com/journals/jamaophthalmology/fullarticle/2778626

8. Sun M, Wadehra M, Casero D, Lin M-C, Aguirre B, Parikh S, Matynia A, Gordon L, Chu A. Epithelial Membrane Protein 2 (EMP2) Promotes VEGF-Induced Pathological Neovascularization in Murine OxygenInduced Retinopathy. Invest Ophthalmol Vis Sci 2020; 61:3.

9. Telander DG, Yu AK, Forward KI, Morales SA, Morse LS, Park SS, Gordon LK. Epithelial Membrane Protein-2 in Human Proliferative Vitreoretinopathy and Epiretinal Membranes. Invest Ophthalmol Vis Sci 2016; 57:3112.

10. Sánchez-Luis E, Joaquín-García A, Campos-Laborie FJ, Sánchez-Guijo F, De las Rivas J. Deciphering Master Gene Regulators and Associated Networks of Human Mesenchymal Stromal Cells. Biomolecules 2020; 10:557.

11. Alvarez MJ, Shen Y, Giorgi FM, Lachmann A, Ding BB, Ye BH, Califano A. Functional characterization of somatic mutations in cancer using network-based inference of protein activity. Nat Genet 2016; 48:838-47.

12. Yu B, Chen L, Zhang W, Li Y, Zhang Y, Gao Y, Teng X, Zou L, Wang Q, Jia H, et al. TOP2A and CENPF are synergistic master regulators activated in cervical cancer. BMC Med Genomics 2020; 13:145.

13. Hwang S-G, Kim K-H, Lee B-M, Moon J-C. Transcriptome analysis for identifying possible gene regulations during maize root emergence and formation at the initial growth stage. Genes Genom 2018; 40:755-66.

14. Doostparast Torshizi A, Wang K. Deconvolution of Transcriptional Networks in Post-Traumatic Stress Disorder Uncovers Master Regulators Driving Innate Immune System Function. Sci Rep 2017; 7:14486.

15. Margolin AA, Nemenman I, Basso K, Wiggins C, Stolovitzky G, Favera RD, Califano A. ARACNE: An Algorithm for the Reconstruction of Gene Regulatory Networks in a Mammalian Cellular Context. BMC Bioinformatics 2006; 7:S7. 
16. Zamora-Fuentes JM, Hernández-Lemus E, Espinal-Enríquez J. Gene Expression and Co-expression Networks Are Strongly Altered Through Stages in Clear Cell Renal Carcinoma. Front Genet 2020; 11:578679.

17. Caporarello N, D’Angeli F, Cambria MT, Candido S, Giallongo C, Salmeri M, Lombardo C, Longo A, Giurdanella G, Anfuso CD, et al. Pericytes in Microvessels: From "Mural" Function to Brain and Retina Regeneration. IJMS 2019; 20:6351.

18. Eyre JJ, Williams RL, Levis HJ. A human retinal microvascular endothelial-pericyte co-culture model to study diabetic retinopathy in vitro. Experimental Eye Research 2020; 201:108293.

19. Yang Y, Huang X, Ma G, Cui J, Matsubara JA, Kazlauskas A, Zhao J, Wang J, Lei H. PDGFR plays an essential role in patient vitreous-stimulated contraction of retinal pigment epithelial cells from epiretinal membranes. Experimental Eye Research 2020; 197:108116.

20. Ma J, Tang W, Gu R, Hu F, Zhang L, Wu J, Xu G. SHP-2-Induced Activation of c-Myc Is Involved in PDGF-B-Regulated Cell Proliferation and Angiogenesis in RMECs. Front Physiol 2020; 11:555006.

21. Krupenko NI, Sharma J, Pediaditakis P, Helke KL, Hall MS, Du X, Sumner S, Krupenko SA. Aldh1I2 knockout mouse metabolomics links the loss of the mitochondrial folate enzyme to deregulation of a lipid metabolism observed in rare human disorder. Hum Genomics 2020; 14:41.

22. Sarret C, Ashkavand Z, Paules E, Dorboz I, Pediaditakis P, Sumner S, Eymard-Pierre E, Francannet C, Krupenko NI, Boespflug-Tanguy O, et al. Deleterious mutations in ALDH1L2 suggest a novel cause for neuro-ichthyotic syndrome. npj Genom Med 2019; 4:17.

23. Dillard C, Kiyohara M, Mah V, McDermott SP, Bazzoun D, Tsui J, Chan AM, Haddad G, Pellegrini M, Chang Y-L, et al. EMP2 Is a Novel Regulator of Stemness in Breast Cancer Cells. Mol Cancer Ther 2020; 19:1682-95.

24. Ruggiero AD, Davis A, Sherrill C, Westwood B, Hawkins GA, Palmer ND, Chou JW, Reeves T, Cox LA, Kavanagh K. Skeletal muscle extracellular matrix remodeling with worsening glycemic control in nonhuman primates. American Journal of Physiology-Regulatory, Integrative and Comparative Physiology 2021; 320: R226-35.

25. Li Y, Jiang S-H, Liu S, Wang Q. Role of IncRNA NEAT1 mediated by YY1 in the development of diabetic cataract via targeting the microRNA-205-3p/MMP16 axis. European Review for Medical and Pharmacological Sciences 2020; 24:5863-70.

26. Li Y, Chen D, Sun L, Wu Y, Zou Y, Liang C, Bao Y, Yi J, Zhang Y, Hou J, et al. Induced Expression of VEGFC, ANGPT, and EFNB2 and Their Receptors Characterizes Neovascularization in Proliferative Diabetic Retinopathy. Invest Ophthalmol Vis Sci 2019; 60:4084. 
27. Liu Y, Dakou E, Meng Y, Leyns L. Loss of Emp2 compromises cardiogenic differentiation in mouse embryonic stem cells. Biochemical and Biophysical Research Communications 2019; 511:173-8.

\section{Figures}

$\mathbf{A}$

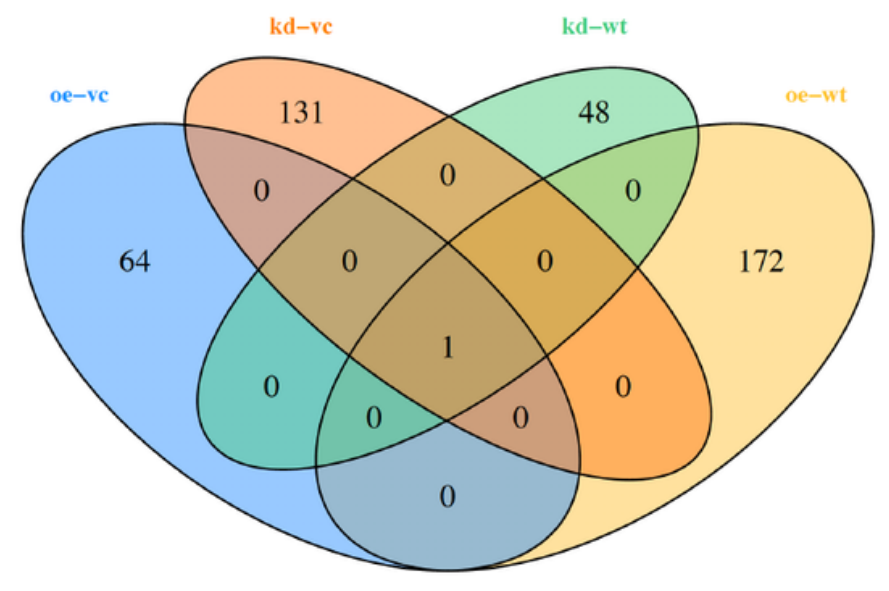

B

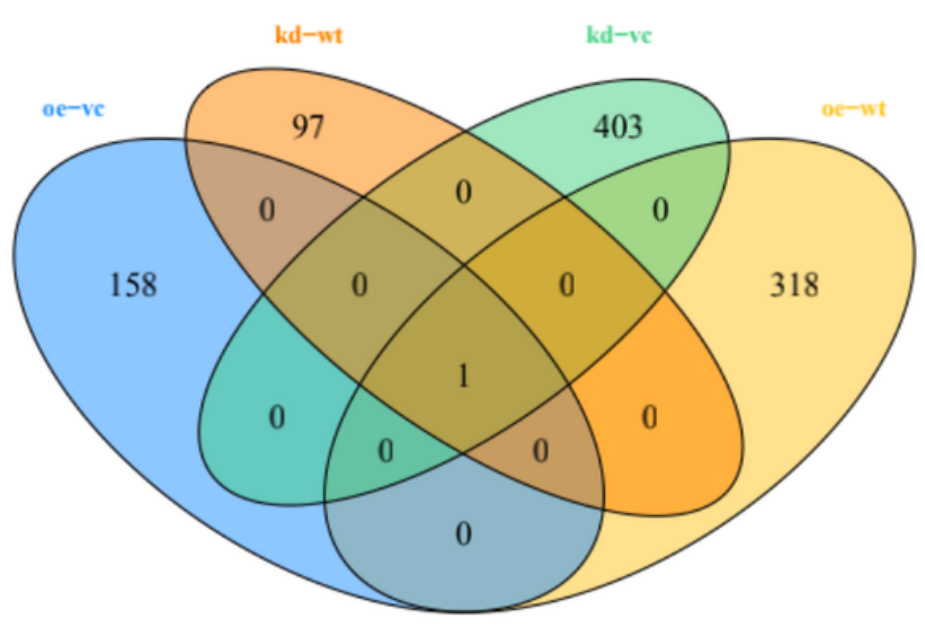

Figure 1

PCA and box plot of all samples All samples had high-quality RNA-seq reads with an average of $96 \%$ reads per sample. Reads with mapping quality score (MAPQ) $<10$ or those with $>5$ mismatches in 100 bp aligned region were discarded. 

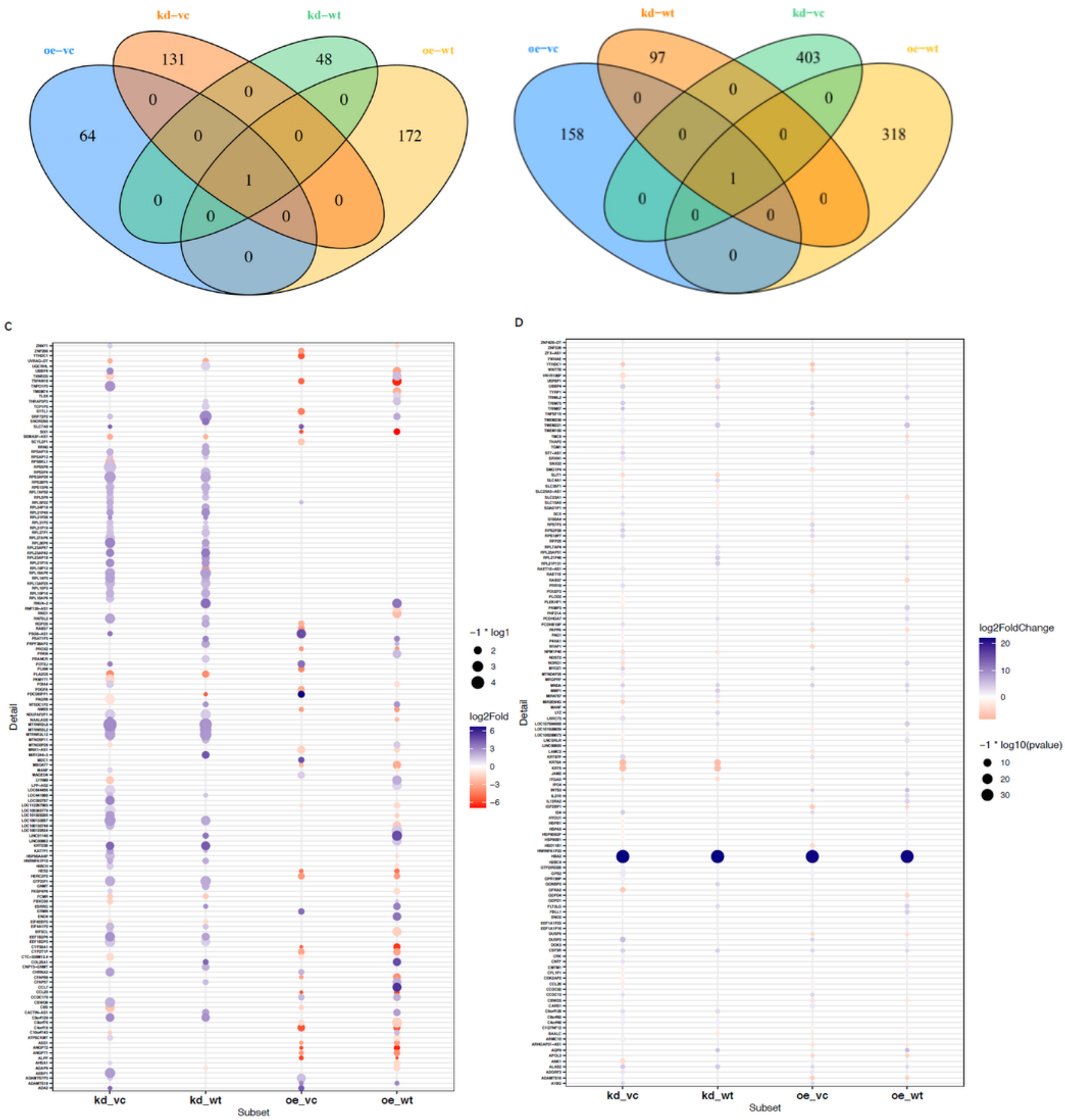

Figure 2

Identification of differential expressed genes(DEGs) in EMP2 regulated RPE cells (A, B) Venn of DEGs in compared groups under hypoxia and normoxia condition, respectively $(C, D)$ Up-and down-regulated genes in compared groups under hypoxia or normoxia condition, respectively DEGs, differentially expressed genes 
A

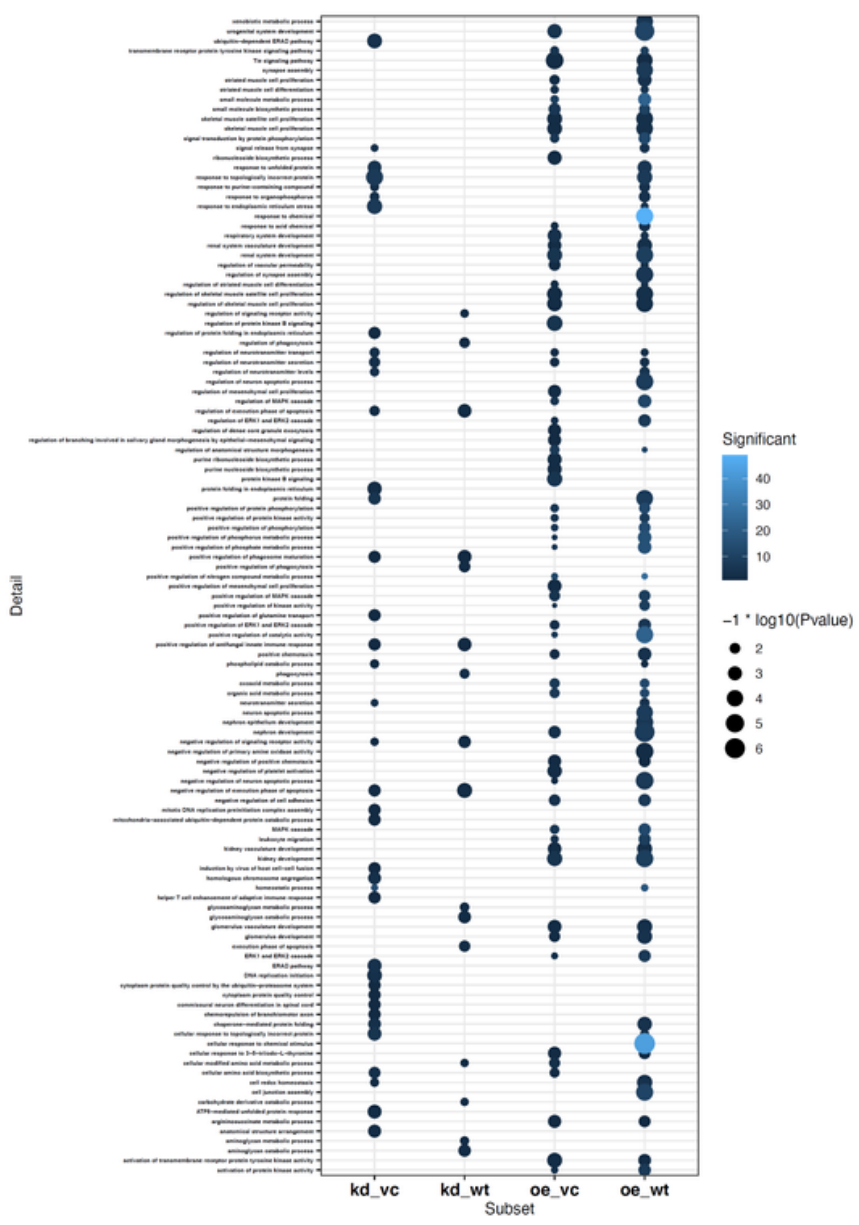

B

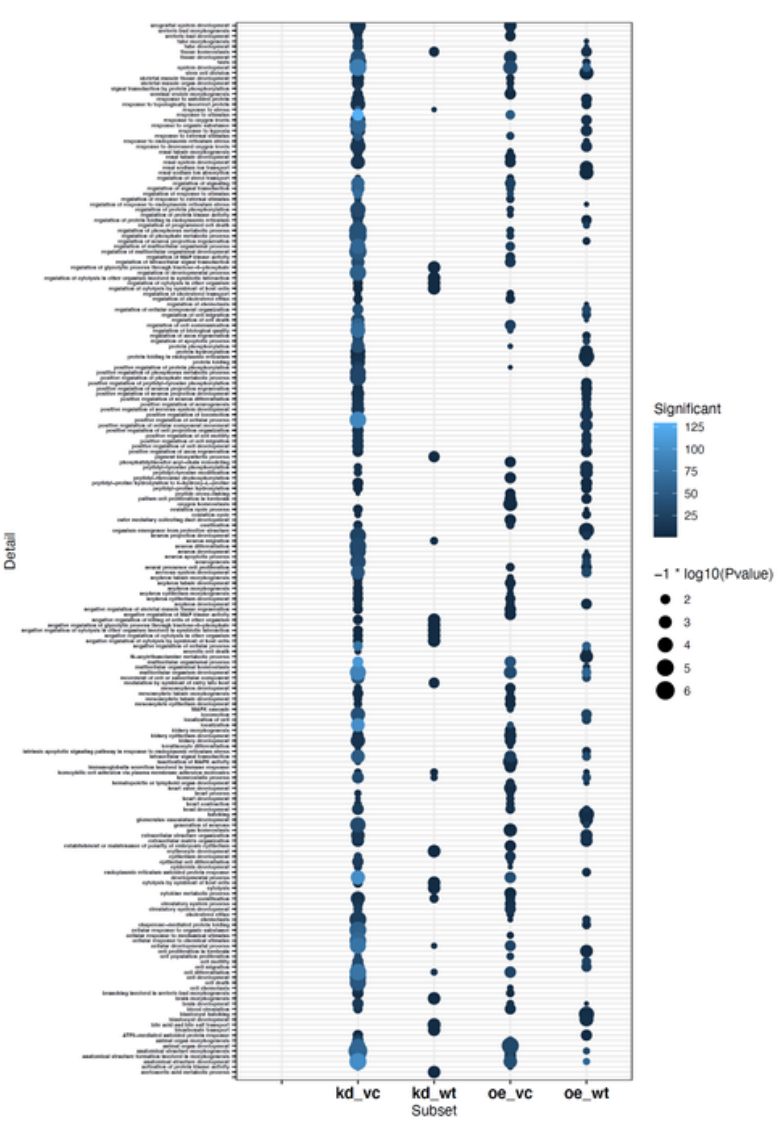

Figure 3

DEGs GO analysis in EMP2 treated RPE cells (A) DEGs Go analysis under hypoxia condition (B) DEGs Go analysis under normoxia 
A

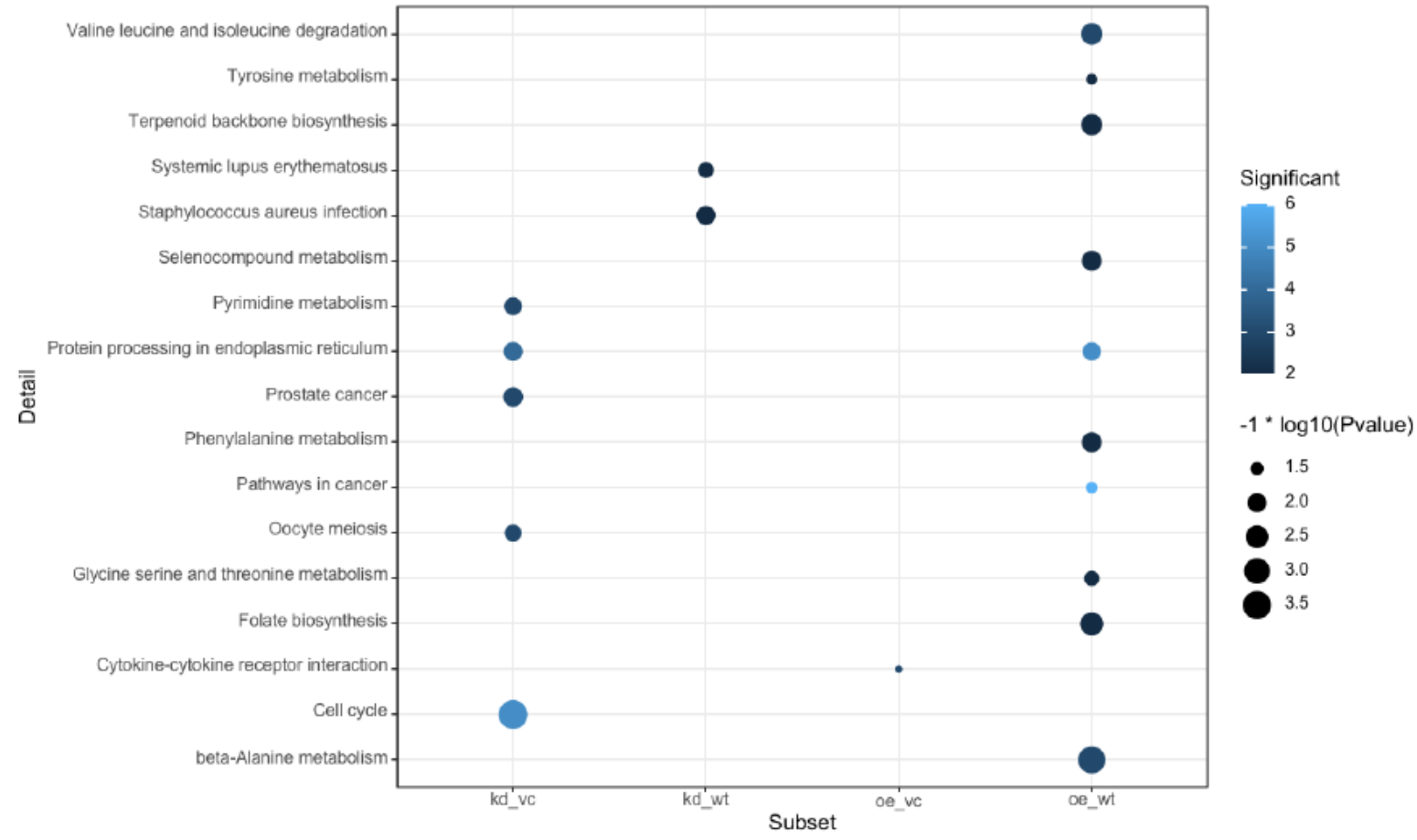

B

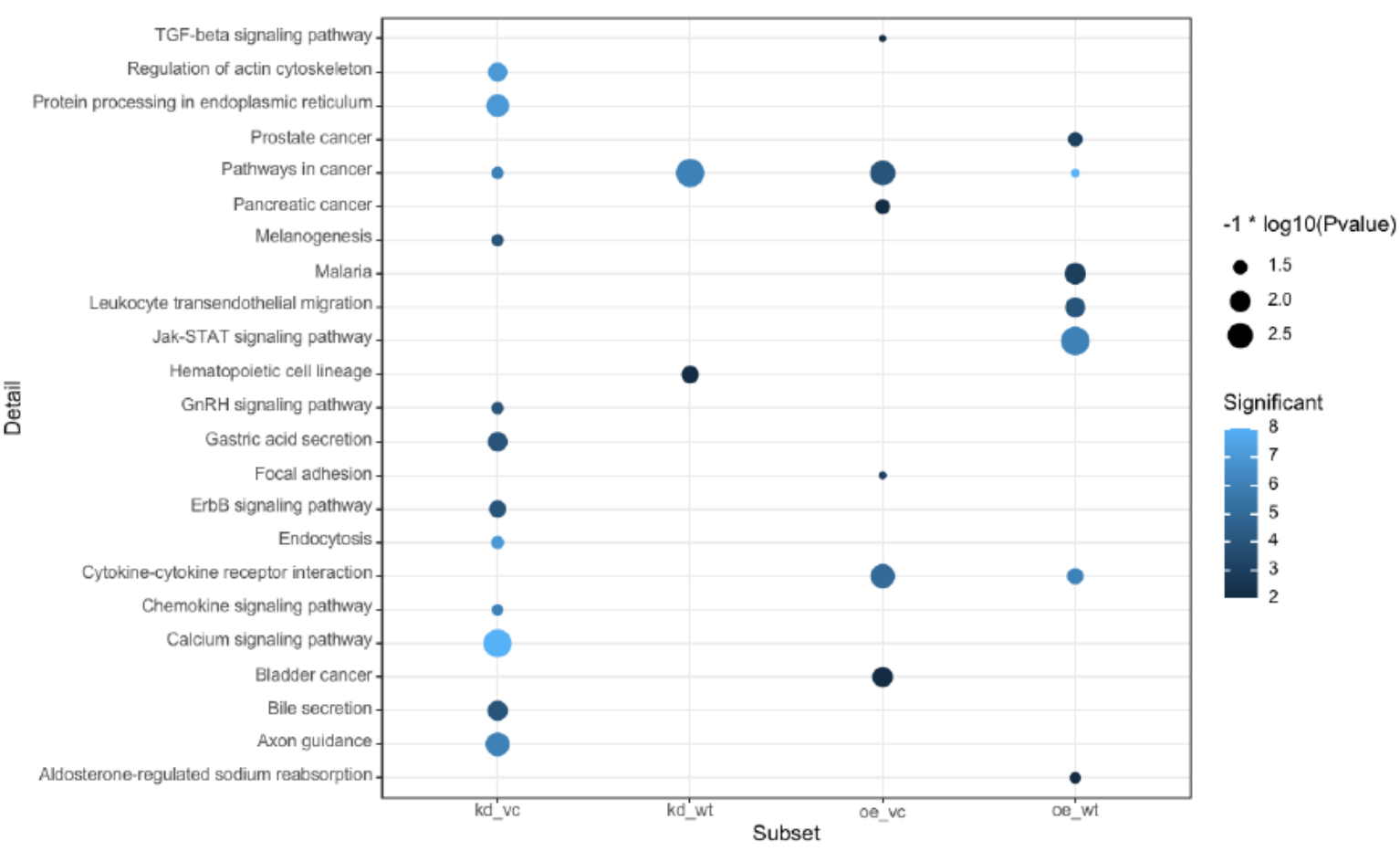

Figure 4

KEGG Pathway analysis of the DEGs (A)KEGG Pathway analysis of DEGs under hypoxia condition (B)KEGG Pathway analysis of DEGs under normoxia condition 


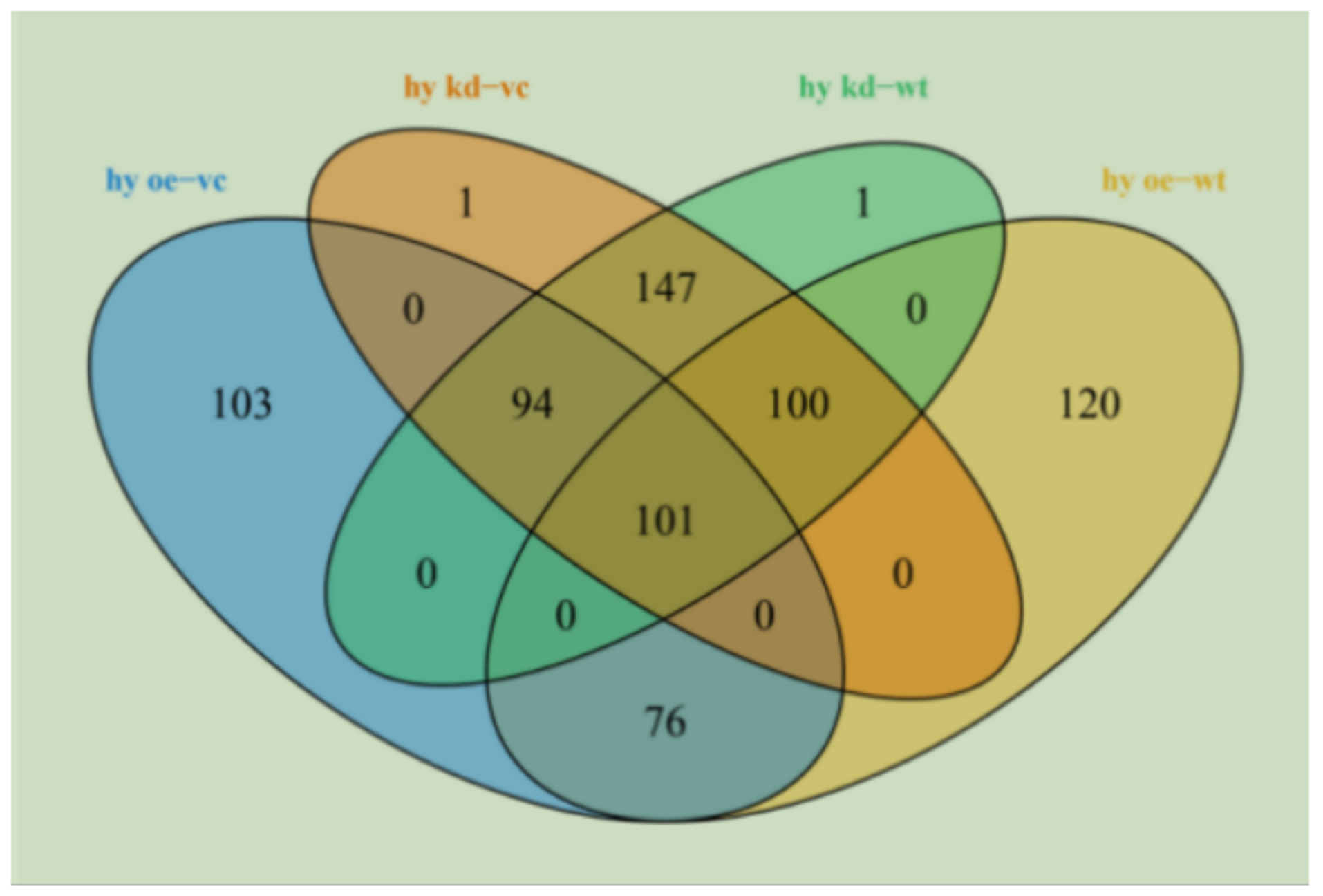

Figure 5

APs of 4 groups under hypoxia Different APs in OE-VC groups were identified when compared to KD-VC groups, as well as APs in OE-WT group when compared to KD-WT group under hypoxia 


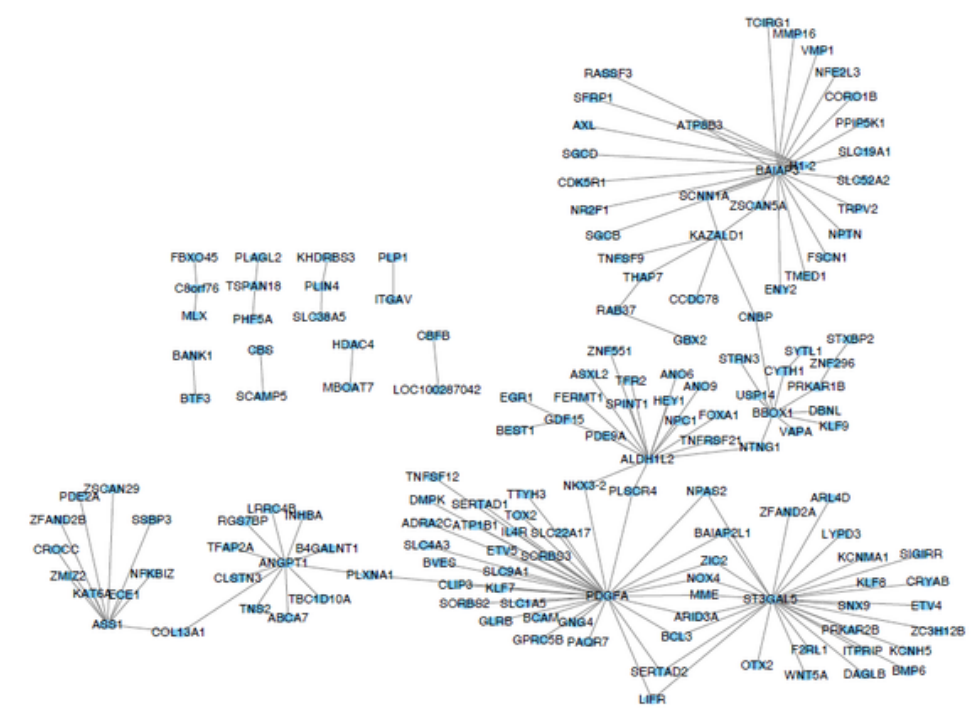

B C
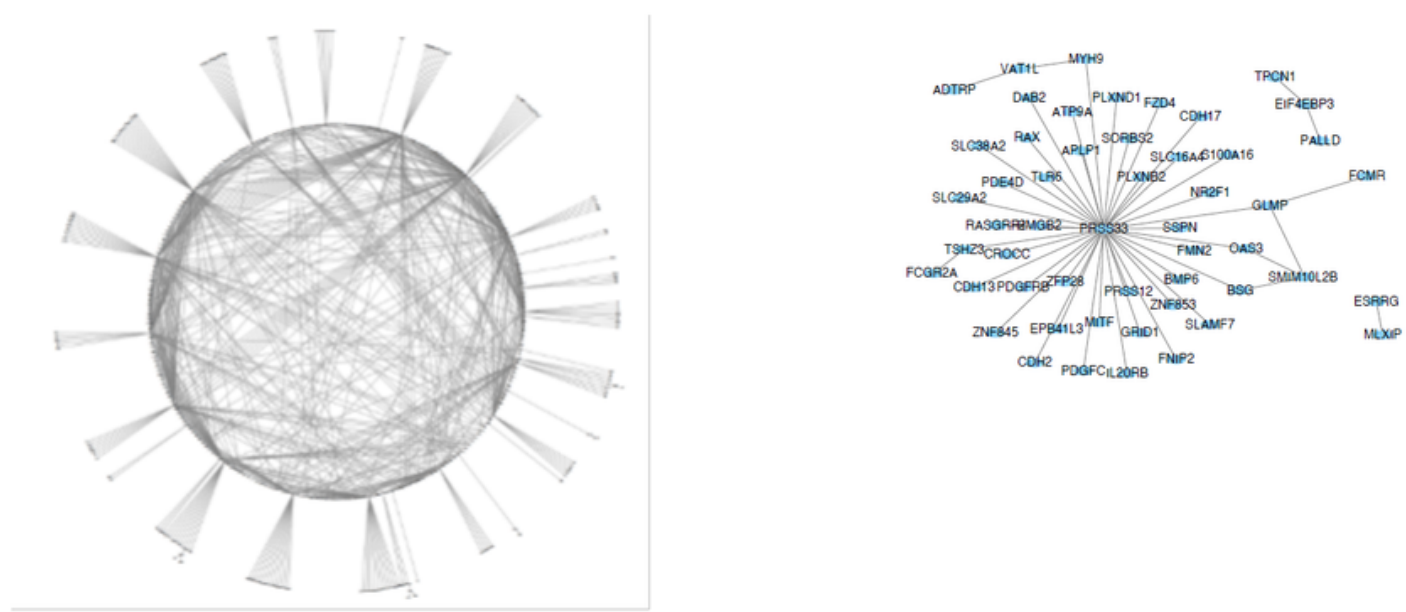

D

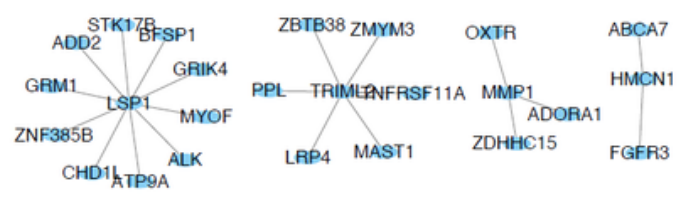

Figure 6

Transcription Regulator network (TRN) of APs and DEGs (A) TRN of hypoxia OE vs VC group, (B)TRN of normoxia OE vs VC group, (C) TRN of hypoxia KD vs WT group, (D)TRN of normoxia KD vs WT group 
This is a list of supplementary files associated with this preprint. Click to download.

- Supplementary.docx 